\title{
Cardiovascular reasons for restricted or refused eligibility in competitive sports among adolescent athletes
}

\author{
Pia Brecht*, Michael Cassel, Claudia Beckendorf and Frank Mayer \\ Department of Sports Medicine, University of Potsdam, Potsdam, Germany
}

\begin{abstract}
Background: General recommendations for the cardiovascular screening procedures required for pre-participation examination (PPE) in adolescent athletes are not available, yet. It is debated, whether the application of ECG and echocardiography to prevent sudden cardiac death (SCD) can be justified due to rare pathological findings in adolescents and consequently low cost-effectiveness.

Purpose: To assess the frequency of pathological cardiovascular findings that lead to a restricted or refused sports-specific eligibility in adolescent athletes.

Methods: Previously recorded data of PPE before entrance in an elite school of sport from 2268 adolescent athletes (965 girls, 1303 boys, age $12.1 \pm 0.6$, BMI 18.4 \pm 2.6 ) out of 18 sports disciplines were analyzed. The PPE consisted of clinical examination, resting and stress ECGs as well as an echocardiography. Cardiovascular abnormalities led to further diagnostics and/or therapeutic recommendations. Depending on the results, eligibility was rated "fully given", "with restriction" or "refused".

Results: Echocardiographies were suspicious in 147 cases (6.5\%). 53 athletes (2.3\%) showed ventricular extrasystoles under stress ECG of which only 25 (1.1\%) resting ECGs were suspicious before. In 19 cases a 24-hours-ECG was indicated.

For two athletes (0.1\%) eligibility was refused due to remaining ASD following surgery in childhood for Fallot tetralogy as well as an ASD (secundum type) in another athlete. Eligibility was restricted in 7 athletes (0.3\%) with left ventricular hypertrophy (1), suspected Long QT syndrome (1), preexcitation syndrome (2), supraventricular extrasystolies (1), and ventricular extrasystolies (2).

Conclusion: Eligibility-restricting cardiovascular findings in adolescent athletes are rare. Nevertheless, prevention of SCD or progression of heart diseases under professional athletic training should be an essential aim of cardiovascular screening in PPE. All restricting and refusing causes in present study wouldn't have been found through medical history and resting ECG alone.
\end{abstract}

\begin{abstract}
Abbreviations: AOd: Aorta Diastolic, ASD: Atrial Septal Defect, BSA: Body surface area, IVSd: Interventricular Septum Diastolic, Las: Left Atrium Systolic, LVDd: Left Ventricle Diastolic, PPE: Pre-Participation Examination, PWd: Posterior Wall, Diastolic, RVDd: Right Ventricle, Diatolic, RVWd: Right Ventricular Wall Diastolic, SCD: Sudden Cardiac Death
\end{abstract}

\section{Introduction}

The main goal of Pre-Participation Examination (PPE) including cardiovascular screening is to minimize the risk of sudden cardiac death (SCD) in inconspicuous athletes. Furthermore, it's intended to estimate harm, professional athleticism can cause to the cardiovascular system especially during growth in adolescents.

There is only low evidence that cardiovascular screening (including resting and exercise ECG and echocardiography) is suitable to identify possible risks for SCD in athletes at a reasonable cost-effectiveness $[1,2]$. It is discussed, that large numbers of false positive findings are concluding in costly additional investigations while apparently healthy screened athletes continue training in a false sense of security [1]. However, it has been previously shown, that cardiovascular abnormalities leading to restricted or refused eligibility are not detectable by clinical examination or resting ECG alone [3].

The implementation of exercise ECG for the diagnostic of cardiovascular disease in clinically healthy athletes lacks evident data.
In consequence management of pathologic findings regarding the athletic career are not defined yet [4]. Clinically, a combination of resting 12-lead-ECG and echocardiography is seen to be an appropriate diagnostic approach to identify most common causes for SCD (e.g. malignant tachyarrhythmias, arrhythmogenic disorders, hypertrophic cardiomyopathy, coronary congenital disorders, channelopathies) or other cardiovascular diseases that aggravate following intensive athletic training $[2,3,5]$. Even though there is no clear recommendation for use of routine echocardiography, it is performed regularly under standardized conditions as part of cardiovascular screening during PPE [6]. As proven in feasibility studies, screening echocardiographies are performed reliably by trained physicians supervised by a consultant with specialization in cardiology $[7,8]$.

The purpose of this study was to assess the frequency of pathological cardiovascular findings through ECG, exercise ECG and echocardiography that lead to a restricted or refused sports-specific

${ }^{\star}$ Correspondence to: Pia Brecht, University Outpatient Clinic, Am Neuen Palais 10, 14469 Potsdam, Germany, E-mail: brecht@uni-potsdam.de

Key words: adolescent athletes, echocardiography, eligibility, pre-participation examination

Received: October 05, 2018; Accepted: October 22, 2018; Published: October 26,2018 
Table 1. Reference values of echocardiographic dimensions in relation to body surface area (BSA) in children and adolescents

\begin{tabular}{|c|c|c|c|c|c|c|c|}
\hline $\operatorname{BSA}\left(\mathrm{m}^{2}\right)$ & RVWd (mm) & RVDd (mm) & IVSDd (mm) & LVDd (mm) & PWd (mm) & Aod $(\mathrm{mm})$ & LAs (mm) \\
\hline 0.25 & $2.6 \pm 1.2$ & $8.7 \pm 4.5$ & $3.8 \pm 1.4$ & $20.0 \pm 3.6$ & $3.6 \pm 1.0$ & $10.4 \pm 2.4$ & $14.0 \pm 3.5$ \\
\hline 0.3 & $2.7 \pm 1.1$ & $8.7 \pm 4.4$ & $3.9 \pm 1.4$ & $22.9 \pm 3.9$ & $4.1 \pm 1.3$ & $11.3 \pm 2.3$ & $15.3 \pm 3.8$ \\
\hline 0.4 & $2.7 \pm 1.1$ & $8.9 \pm 4.5$ & $4.1 \pm 1.5$ & $26.0 \pm 5.0$ & $4.2 \pm 1.3$ & $12.9 \pm 2.0$ & $16.8 \pm 3.8$ \\
\hline 0.5 & $2.8 \pm 1.1$ & $9.3 \pm 4.5$ & $4.3 \pm 1.6$ & $29.0 \pm 5.6$ & $4.6 \pm 1.5$ & $14.9 \pm 2.7$ & $18.7 \pm 4.2$ \\
\hline 0.6 & $2.8 \pm 1.1$ & $9.6 \pm 4.4$ & $4.8 \pm 1.5$ & $31.6 \pm 5.6$ & $4.8 \pm 1.5$ & $15.6 \pm 2.8$ & $20.1 \pm 4.0$ \\
\hline 0.7 & $2.8 \pm 1.1$ & $10.1 \pm 4.4$ & $5.0 \pm 1.5$ & $33.9 \pm 6.5$ & $5.2 \pm 1.7$ & $16.9 \pm 3.4$ & $21.1 \pm 5.0$ \\
\hline 0.8 & $2.8 \pm 1.1$ & $10.5 \pm 4.7$ & $5.2 \pm 1.6$ & $35.8 \pm 6.2$ & $5.7 \pm 2.1$ & $17.9 \pm 3.4$ & $22.5 \pm 6.0$ \\
\hline 0.9 & $2.8 \pm 1.1$ & $11.0 \pm 4.6$ & $5.6 \pm 1.8$ & $37.1 \pm 6.1$ & $5.9 \pm 2.2$ & $18.7 \pm 3.6$ & $23.2 \pm 6,2$ \\
\hline 1.0 & $2.8 \pm 1.1$ & $11.2 \pm 4.8$ & $5.8 \pm 1.8$ & $38.5 \pm 6.8$ & $5.9 \pm 2.2$ & $19.9 \pm 3.6$ & $25.0 \pm 5.8$ \\
\hline 1.1 & $2.9 \pm 1.1$ & $11.8 \pm 4.4$ & $6.2 \pm 1.9$ & $39.4 \pm 6.9$ & $6.3 \pm 2.4$ & $20.9 \pm 3.4$ & $25.2 \pm 5.7$ \\
\hline 1.2 & $2.9 \pm 1.1$ & $12.4 \pm 4.8$ & $6.5 \pm 1.8$ & $41.7 \pm 6.2$ & $6.6 \pm 2.5$ & $21.0 \pm 3.5$ & $26.0 \pm 5.1$ \\
\hline 1.3 & $3.0 \pm 1.1$ & $13.5 \pm 5.0$ & $6.6 \pm 1.8$ & $42.4 \pm 6.6$ & $6.9 \pm 2.6$ & $21.7 \pm 4.2$ & $27.3 \pm 5.6$ \\
\hline 1.4 & $3.0 \pm 1.1$ & $14.0 \pm 5.0$ & $6.7 \pm 1.8$ & $43.3 \pm 6.0$ & $6.9 \pm 2.6$ & $22.7 \pm 4.8$ & $28.2 \pm 5.4$ \\
\hline 1.5 & $3.1 \pm 1.2$ & $15.6 \pm 5.6$ & $7.4 \pm 2.2$ & $45.4 \pm 6.4$ & $7.7 \pm 2.8$ & $23.6 \pm 5.4$ & $29.9 \pm 6.2$ \\
\hline 1.75 & $3.1 \pm 1.2$ & $16.5 \pm 6.2$ & $8.0 \pm 2.4$ & $46.8 \pm 6.0$ & $8.1 \pm 3.0$ & $24.4 \pm 6.2$ & $30.4 \pm 6.6$ \\
\hline 2.0 & $3.1 \pm 1.2$ & $17.5 \pm 6.0$ & $8.3 \pm 2.5$ & $53.4 \pm 8.0$ & $8.1 \pm 3.0$ & $27.4 \pm 4.4$ & $32.5 \pm 8.8$ \\
\hline
\end{tabular}

eligibility in adolescent athletes due to high probability of worsening or potential risk of SCD.

\section{Subjects and methods}

2268 adolescent athletes ( 965 girls, 1303 boys, age $12.1 \pm 0.6$, from 18 sports disciplines, BMI $18.4 \pm 2.6$ ) were included, undergoing a PPE before enrollment to an elite sports school.

As part of the PPE both a 12-lead-ECG and an echocardiography were performed and evaluated under standardized criteria following recommendations by Scharhag and Burstaller [9] based on the Seattle Criteria and the American Society of Echocardiography [10]. Seattle Criteria as special ECG evaluation criteria for athletes were considered due to electromorphological training adaptions in athletes' heart that differ from the normal population [11,12]. In echocardiography's LV inner diastolic diameter, posterior wall thickness, septal thickness, left atrial and aortic root diameters and right ventricular inner diameter were all measured in M-Mode and valued depending on body surface area (Table 1) [13]. Valves were evaluated morphologically and functionally, as well as the left ventricular systolic and diastolic function. Additionally, an exercise ECG, using a stepwise incremental protocol, was performed on a cycle ergometer. Exercise ECG was evaluated as suspicious when a relevant number of ventricular ectopic beats occurred.

\section{Results}

Abnormal resting ECGs were found in 50 athletes (2.2\%), suspicious exercise ECGs occurred in 53 (2.3\%) athletes of which 25 (1.1\%) showed a suspicious resting ECG before. In 19 cases a 24-hoursECG was indicated.

Echocardiographies were suspicious in 147 cases (6.5\%). Mild valve insufficiencies without clinical manifestation or morphological anomaly were the main findings and led to a recommendation of a follow-up examination after one or two years but never resulted in a restriction of eligibility.

Eligibility was refused in 2 athletes (0.1\%), and in 7 cases $(0.3 \%)$ it was restricted. Reasons for refused eligibility were childhood surgery after Fallot tetralogy with remaining ASD as well as an ASD (secundum type) in another athlete, solely apparent through echocardiography. Reasons for restricted eligibility were left ventricular hypertrophy
(1), suspected Long QT syndrome (1), preexcitation syndrome (2), supraventricular extrasystolies (1), and ventricular extrasystolies (2). All of the findings leading to restriction were detected by additionally using exercise ECG and/or echocardiography. In all cases of refusal or restriction a second opinion presentation to a pediatric cardiologist was recommended. In two electrophysiological testing possibly followed by ablation has been indicated. Restricted eligibility in 2 athletes followed after suspicious exercise ECG and 24-hours-ECG because of extrasystoles.

\section{Discussion}

In line with previous data [6] the rate of refused or restricted eligibility for competitive sports due to cardiovascular reasons in adolescent athletes was low. However, relevant cardiovascular diseases including the most common causes for SCD, whose prevention is one of the main goals of cardiovascular screening in PPE, are solely detectable by an additional combination of resting and exercise ECG and echocardiography. The pathological echocardiographic findings leading to restricted and/or refused eligibility were not detected by ECG abnormalities alone at rest or exercise. Vice-versa, supported by the addition of echocardiography, some ECG abnormalities didn't show morphologic or functional pathologies of the heart.

Performed under standardized conditions, echocardiography is a helpful and indispensable additional tool to identify cardiovascular abnormalities. Furthermore, it reduces false-negative and false-positive ECG findings. This is essential to not exclude an adolescent athlete from physical activity without evidence-based reason.

\section{Funding}

National grant, local ministry.

\section{Competing Interest}

None.

\section{References}

1. Mosterd A (2018) Pre-participation examination screening of asymptomatic athletes. Neth Heart J 26: 123-126. [Crossref]

2. Menafoglio A (2014) Costs and yield of a 15-month preparticipation cardiovascular examination with ECG in 1070 young athletes in Switzerland: Implications for routine ECG screening. Br J Sports Med 48: 1157-1161. [Crossref] 
3. Mont L, Pelliccia A, Sharma S, Biffi A, Borjesson M et al. (2017) Pre-participation cardiovascular evaluation for athletic participants to prevent sudden death: Position paper from the EHRA and the EACPR, branches of the ESC. Endorsed by APHRS, HRS, and SOLAECE. Eur J Prev Cardiol 24: 41-69. [Crossref]

4. van de Sande DA, Breuer MA, Kemps HM (2016) Utility of Exercise Electrocardiography in Pre-participation Screening in Asymptomatic Athletes: A Systematic Review. Sports Med 46: 1155-1164. [Crossref]

5. Calò L, Sperandii F, Martino A, Guerra E, Cavarretta E, et al. (2015) Echocardiographic findings in 2261 peri-pubertal athletes with or without inverted $\mathrm{T}$ waves at electrocardiogram. Heart 101: 193-200. [Crossref]

6. Mayer F, Bonaventura K, Cassel M, Mueller S, Weber J, et al. (2012) Medical results of preparticipation examination in adolescent athletes. Br J Sports Med 46: 524-530. [Crossref]

7. Kerkhof D, Gleason CN, Basilico FC, Corrado GD (2016) Is There a Role for Limited Echocardiography During the Preparticipation Physical Examination? PMR 8: S36-44. [Crossref]
8. Asplund CA, Asif IM (2014) Cardiovascular preparticipation screening practices of college team physicians. Clin J Sport Med 24: 275-279. [Crossref]

9. Scharhag J, Burgstahler C (2013) The Sports ECG: Current interpretations and recommendations. DGSP 64: 352-356.

10. Lang RM, Badano LP, Mor-Avi V, Afilalo J, Armstrong A, et al. (2015) Recommendations for Cardiac Chamber Quantification by Echocardiography in Adults: An Update from the American Society of Echocardiography and the European Association of Cardiovascular Imaging. J Am Soc Echocardiogr 28: 1-39. [Crossref]

11. Pickham D, Hsu D, Soofi M, Goldberg JM, Saini D, et al. (2016) Optimizing QT Interval Measurement for the Preparticipation Screening of Young Athletes. Med Sci Sports Exerc 48: 1745-1750. [Crossref]

12. Riding NR, Sheikh N, Adamuz C, Watt V, Farooq A, et al. (2015) Comparison of the three current sets of electrocardiographic interpretation criteria for use in screening athletes. Heart 101: 384-390. [Crossref]

13. Gutberlet M (2017) Normwerte und Formeln fuer die Echokardiografie in Bildgebende Diagnostik angeborender Herzfehler, Thieme Verlagsgruppe, Stuttgart.

Copyright: $\odot 2018$ Brecht P. This is an open-access article distributed under the terms of the Creative Commons Attribution License, which permits unrestricted use, distribution, and reproduction in any medium, provided the original author and source are credited. 